\title{
Measurement of Local Scale Wave Transformation Effects at NCEX Using AROSS Imagery
}

\author{
J. Zandy Williams and John Dugan \\ Areté Associates \\ 1725 Jefferson Davis Hwy, Suite 703 \\ Arlington, VA 22202 \\ phone: (703) 413-0290 fax: (703) 413-0295 email: williams@arete-dc.com \\ K. Todd Holland and James Kaihatu \\ Naval Research Laboratory \\ Code 7440.3 Building 2438 \\ Stennis Space Center, MS 39529 \\ phone: (228) 688-5320 fax: (228) 688-4476 email: tholland@nrlssc.navy.mil \\ Contract Number: N00014-02-C-0067, Document Number: N00014-02-WX-20523 \\ http://science.whoi.edu/PVLAB/NCEX/wp-lg-scale-video.html
}

\section{LONG-TERM GOALS}

This work seeks an improved understanding of shoaling-wave phenomenology, particularly in areas of complex bathymetry, through the use of novel, large-area, high-resolution, remote-sensing approaches.

\section{OBJECTIVES}

This effort will study the transformation of deep-water waves to the surf-zone as they shoal over a large spatial domain with complex bathymetry. The investigation will compare wave model output parameters, such as significant wave height and period as well as directional spectra, with the values obtained from an airborne, electro-optical, remote sensor. Data will also be used for the investigation of propagation and surf-zone physics with emphasis on the evaluation of the importance of reflection and diffraction effects.

\section{APPROACH}

This effort will use the Airborne Remote Optical Spotlight System (AROSS) at the Nearshore Canyon Experiment (NCEX) to provide remotely-sensed, electro-optical data of shoaling waves from deep water through the surf zone. NCEX is a multi-institutional experiment that will be conducted in Fall 2003 near La Jolla, CA. AROSS is a compact, turret-based optical system that has been designed and constructed for imaging ocean waves from a small, specialized aircraft. It consists of a digital camera, IMU, GPS receiver, and control system mounted on a single-engine aircraft. The system was designed to spotlight or stare at a specific geographic location for up to several minutes and collect time series of images along with precise navigational and pointing data. By mapping the imagery to a geodetic grid at the level of the mean ocean surface, the space-time characteristics of the waves can be extracted. Data cubes of information (two spatial dimensions plus time) will be available from AROSS overflights with durations of up to 4 hour over multiple coverage areas of up $3 \times 3 \mathrm{~km}$. These measurements are necessary to properly characterize the transformation of deep-water waves to the 


\section{Report Documentation Page}

Form Approved

OMB No. 0704-0188

Public reporting burden for the collection of information is estimated to average 1 hour per response, including the time for reviewing instructions, searching existing data sources, gathering and maintaining the data needed, and completing and reviewing the collection of information. Send comments regarding this burden estimate or any other aspect of this collection of information,

including suggestions for reducing this burden, to Washington Headquarters Services, Directorate for Information Operations and Reports, 1215 Jefferson Davis Highway, Suite 1204, Arlington

VA 22202-4302. Respondents should be aware that notwithstanding any other provision of law, no person shall be subject to a penalty for failing to comply with a collection of information if it

does not display a currently valid OMB control number.

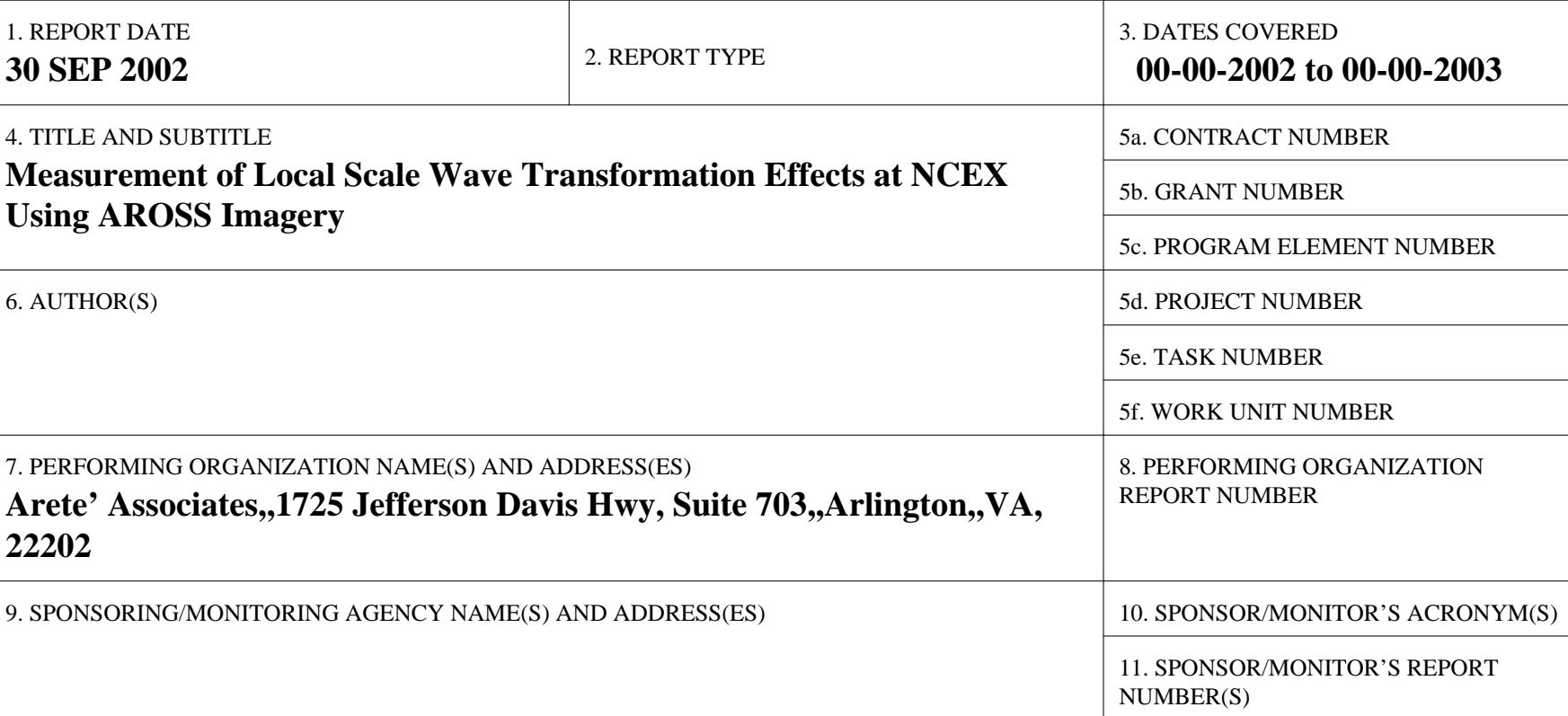

12. DISTRIBUTION/AVAILABILITY STATEMENT

Approved for public release; distribution unlimited

13. SUPPLEMENTARY NOTES

14. ABSTRACT

This work seeks an improved understanding of shoaling-wave phenomenology, particularly in areas of complex bathymetry, through the use of novel, large-area, high-resolution, remote-sensing approaches.

15. SUBJECT TERMS

16. SECURITY CLASSIFICATION OF:

a. REPORT

unclassified b. ABSTRACT

unclassified c. THIS PAGE

unclassified
17. LIMITATION OF ABSTRACT

Same as

Report (SAR)
18. NUMBER OF PAGES

6 19a. NAME OF

RESPONSIBLE PERSON 
surf-zone over the complicated bathymetry present at the experiment site. Imagery from AROSS will cover the entire shoaling region, as well as the surf, providing data suitable for deriving directional spectra at multiple locations over the NCEX area. These spectra can be compared to calculations from shoaling-wave models to aid the identification and investigation of the relative importance of physics not represented in the models. Algorithms developed for extracting surf-zone parameters from towerbased video will also be applied to AROSS imagery in near-real time and the nowcasts from this analysis will be made available to NCEX investigators.

\section{WORK COMPLETED}

In preparing for participation of AROSS in NCEX, an algorithm to determine the modulation-transferfunction (MTF) to convert measured slope radiance to waveheight has been developed. Images of the ocean surface measure the radiance that is emitted and reflected at the surface. At modest grazing angles outside the surf zone, the modulations in the radiance are dominated by the reflection of the sky radiance by the changing wave slopes (Walker 1994). Since the wavelengths of visible light are much shorter than the lengths of the waves on the surface, the reflection mechanism is mostly specular and the measured radiance modulations closely replicate the line-of-sight wave slope modulations. The precise magnitude of the radiance modulations depends upon the wave slopes and the radiance gradient of the background sky. The latter typically is not known in absolute units nor is the quantification effect of the intervening atmosphere. However, an estimate of the radiance-to-slope transfer function can be estimated using the relation (Stillwell 1974):

$$
L_{\text {total }}=L_{\text {path }}+\tau\left(L_{\text {upwelling }}+r L_{\text {downwelling }}\right)=L_{\text {path }}+\tau L_{w}
$$

where $L_{\text {total }}$ is the total radiance seen by the sensor, $L_{\text {path }}$ is the path radiance between the sensor and the ocean surface, $L_{\text {upwelling }}$ is the emitted radiance from the sea surface $L_{\text {downwelling }}$ is the radiance incident on the sea surface, $\tau$ is transmittance of the intervening atmosphere and $r$ is the Fresnel reflectivity. The linear terms of a Taylor expansion of (1) in surface-slope angle, $\theta$, yield:

$$
\Delta L_{\text {total }} \approx \Delta \theta\left(\frac{d L_{\text {path }}}{d \theta}+\tau \frac{d L_{w}}{d \theta}\right)
$$

If path radiance is assumed to be negligible and the transmittance is set equal to 1 , then (2) reduces to:

$$
\Delta \theta \approx \Delta L_{\text {total }} / \frac{d L_{w}}{d \theta}
$$

An estimate of waveheight is obtained from integrating the slopes obtained from (3) for images with orthogonal look directions.

Other preparation work include improvements to the image mapping techniques for regions with sparse or variable ground control points and an initial investigation of the sensitivity of models to parameter inputs. 


\section{RESULTS}
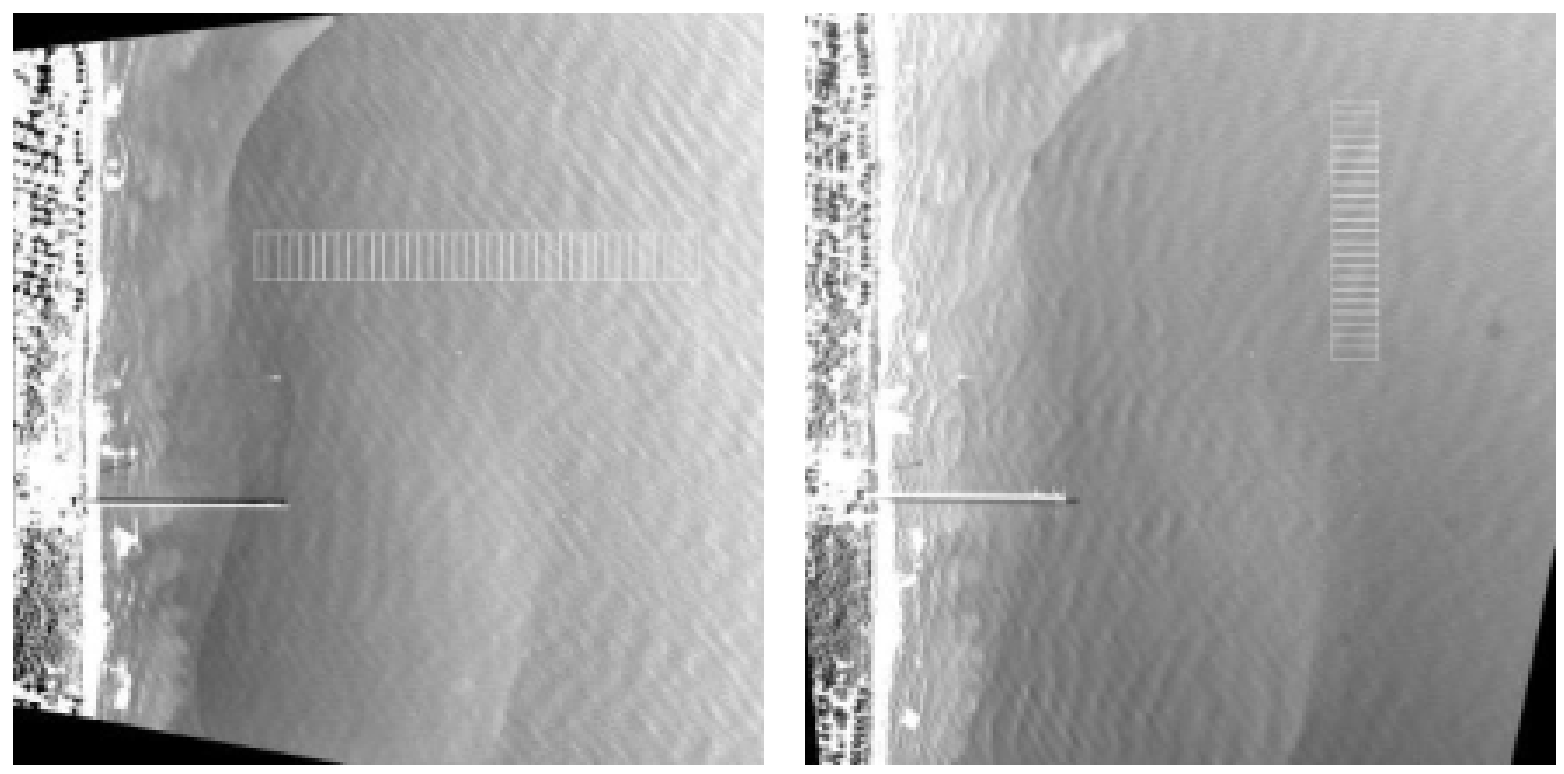

Figure 1. AROSS Imagery from SHOWEX on Nov 4, 1999

[the left image is a cross-shore look and the right image is an along-shore look; the boxes indicate the portions of the imagery used to obtain the radiance gradients for estimating the MTF]

Two images, which are a sample data set used to develop the AROSS MTF, are shown in Figure 1. These images have cross-shore and along-shore look directions and are from the SHOWEX data collection on Nov 4, 1999. The MTF algorithm, represented by Equation (3), was applied to the image gradients, shown in Figure 2, obtained from the regions indicated in Figure 1. The waveheight estimate was obtained by adding in quadrature the results from the two gradients. The estimated waveheight of $0.53 \mathrm{~m}$ compares well with the in situ measurement of $0.58 \mathrm{~m}$.
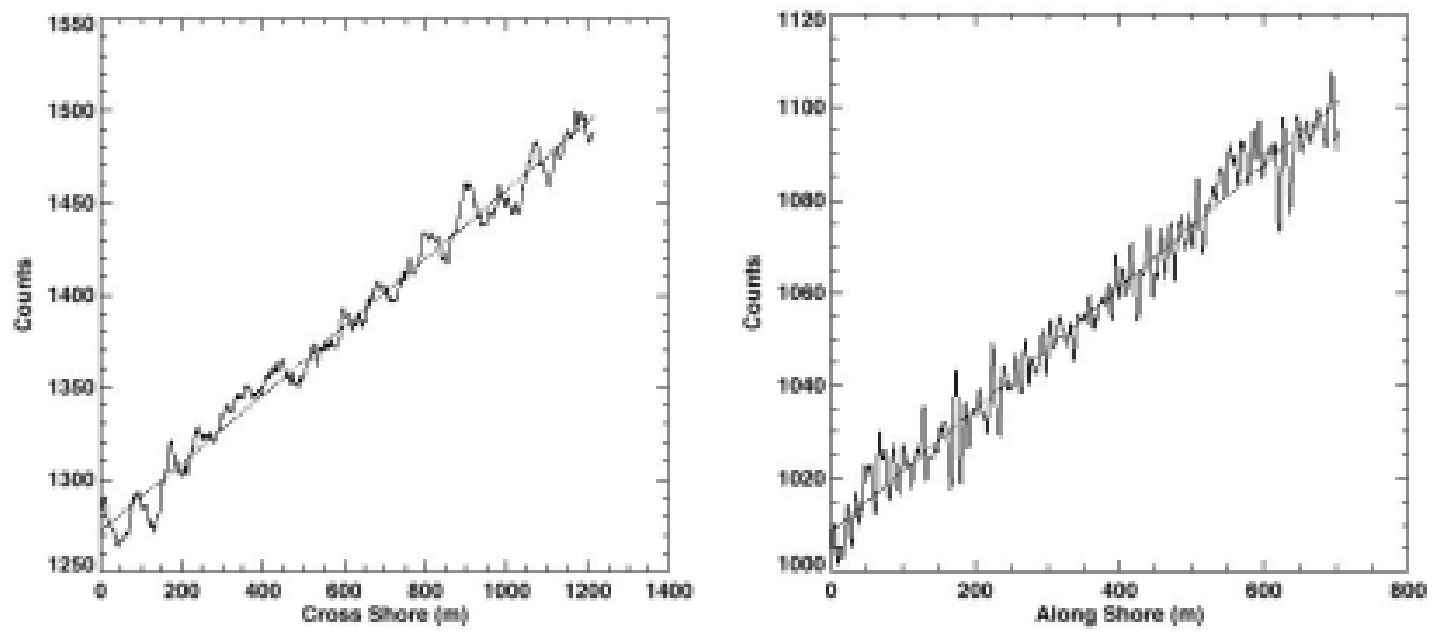

Figure 2. Slope gradients for MTF estimation

[left graph: 1270 counts at $0 \mathrm{~m}, 1380$ counts at $600 \mathrm{~m}, 1490$ counts at $1200 \mathrm{~m}$ ] [right graph: 1010 counts at $0 \mathrm{~m}, 1060$ counts at $400 \mathrm{~m}, 1100$ counts at $750 \mathrm{~m}$ ] 
An investigation of the sensitivity of the waveheights at the NCEX site on offshore spectral parameters has been performed. Using the Simulating Waves Nearshore (SWAN) model (Booij et al. 1999), the study found that misidentification of the peak direction of the input spectrum is the largest contributor to the error of the waveheights nearshore. This error is particularly large in the region where in situ measurements will be performed. Figure 3 shows results for a variation in the initial swell direction of $2.5^{\circ}$ (top) and $7.5^{\circ}$ (bottom). The results indicate how the wave energy is focused and show a change in the shoaled waveheights as a function of spectral direction. This effect can have a big impact for nearshore models that get initial conditions from a large-scale model, such as the Wave Model (WAM; Komen et al. 1994) which has a $\pm 7.5^{\circ}$ uncertainty in peak direction, or from a buoy which has a $\pm 2.5^{\circ}$ degree error. The study also looked waveheight errors due to errors in peak frequency as well as frequency and directional spreading parameters. The results found that these errors were not as large as the errors due to misidentification of direction.

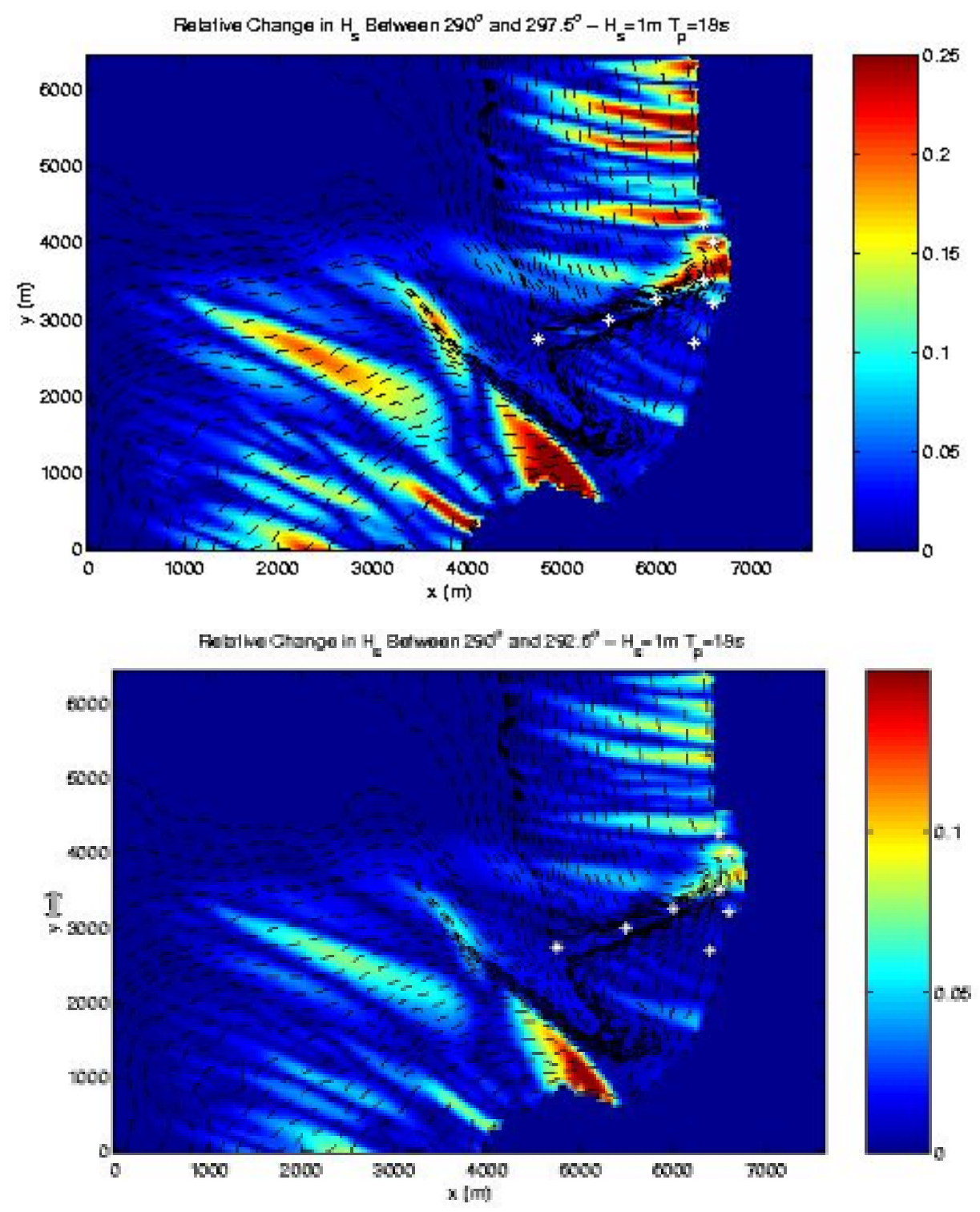

Figure 3: Relative waveheight change at NCEX due to small changes in incident swell direction [graph: upper graph is waveheight change due to $2.5^{\circ}$ change in incident swell peak direction ] [graph: lower graph is waveheight change due to $7.5^{\circ}$ change in incident swell peak direction ] 


\section{IMPLICATIONS/APPLICATIONS}

With the emphasis of the ONR Coastal Dynamics Programs on model-driven experiments, AROSS has the potential for a unique contribution to this effort. In situ measurements provide detailed information of local nearshore phenomena at discrete points in space. These measurements are often used to validate shoaling models, which are initialized with deep-water measurements or large-scale propagation model output. The validation offers no further information concerning the performance of the model elsewhere in space. Data collected by AROSS will provide medium scale measurements and effectively fill the information gap between the two extremes. A self-consistent set of bathymetry, currents, and directional spectra over the entire shoaling region can be retrieved from AROSS data for the evaluation and development of shoaling-wave models.

\section{TRANSITIONS}

None

\section{RELATED PROJECTS}

Areté Associates is developing a new EO imaging system, based on the Airborne Optical Spotlighting System (AROSS) design, that will collect time-series imagery of the ocean surface suitable for scientific research. The new system is a multi-channel AROSS designed for low-cost production, through the use of commercial-off-the-shelf (COTS) components, and low-cost operation, through the use of commercial aerial photography airplanes. This work is funded under the SBIR Phase II contract N00014-02-C-0183 for Solicitation Topic N01-035, "Four Dimensional (4-D) Atmospheric and Oceanographic Instrumentation". Areté was awarded this contract based on a proposal entitled, "A Low-Cost Airborne EO Oceanographic Measurement System”.

Findings and algorithms improvements resulting from this effort will be incorporated into the NRL Littoral Environmental Nowcasting System (LENS) program that seeks to use remotely derived information to drive state-of-the-art models of littoral dynamics.

\section{REFERENCES}

Booij, N., Ris, R.C., and Holthuijsen, L.H. 1999. A third generation wave model for coastal regions. J. Geophys. Res., 104, 7649-7666.

Komen, G.J., Cavaleri, L., Donelan, M.A., Hasselmann, K., Hasselmann, S., and Janssen, P.A.E.M. 1994. Dynamics and Modeling of Ocean Waves, Cambridge University Press, Cambridge, U.K., 532 p.

R..E. Walker, Marine Light Field Statistics, John Wiley \& sons, Inc. (1994)

D. Stilwell, Jr and R.O. Pilon, J. Geophys. Res., Vol 79, \#9, 1974, 1277-1284 